Rhode Island College

Digital Commons @ RIC

Master's Theses, Dissertations, Graduate

Master's Theses, Dissertations, Graduate Research and Major Papers Overview

Research and Major Papers

$1-1-2014$

\title{
Timelines of Oral Care and Early-Onset Ventilator Associated Pneumonia Prevention
}

Kristen M. Francoeur

Rhode Island College

Follow this and additional works at: https://digitalcommons.ric.edu/etd

Part of the Other Nursing Commons, and the Other Public Health Commons

\section{Recommended Citation}

Francoeur, Kristen M., "Timelines of Oral Care and Early-Onset Ventilator Associated Pneumonia Prevention" (2014). Master's Theses, Dissertations, Graduate Research and Major Papers Overview. 234. https://digitalcommons.ric.edu/etd/234

This Major Paper is brought to you for free and open access by the Master's Theses, Dissertations, Graduate Research and Major Papers at Digital Commons @ RIC. It has been accepted for inclusion in Master's Theses, Dissertations, Graduate Research and Major Papers Overview by an authorized administrator of Digital Commons @ RIC. For more information, please contact digitalcommons@ric.edu. 
Kristen M. Francoeur

A Major Paper Submitted in Partial Fulfillment

of the Requirements for the Degree of

Master of Science in Nursing

in

The School of Nursing

Rhode Island College 


\begin{abstract}
Hospital-acquired infections, including ventilator associated pneumonia (VAP), are a significant cause of morbidity and mortality and associated with increased costs and length of stay (Chastre \& Fagon, 2002; NNIS, 2004). Ventilator associated pneumonia is believed to primarily result from aspiration of oropharyngeal secretions around the endotracheal tube cuff into the lungs (Grap, Munro, Unoki, Hamilton, \& Ward, 2012). A randomized control trial tested early application of oral chlorhexidine $(\mathrm{CHG})$ on oral microbial flora and VAP in trauma patients and suggested that early (within 12 hours of intubation) application may reduce VAP rates in trauma patients (Grap, Munro, Hamilton, Elswick, Sessler \& Ward, 2011). The VAP rate in a local Level 1 trauma center, 11-bed trauma intensive care unit (TICU) was 8.7 per 1000 device days, above the national average (NHSN, 2011). The purpose of this research was to explore the relationship between the time of insertion of an endotracheal tube and first $\mathrm{CHG}$ application and early onset (within 72 hours of intubation) VAP. A retrospective chart review of the records of randomly selected adult intubated trauma patients hospitalized on the TICU was conducted. Collected data included: time of intubation; timing of CHG application; VAP occurrences; and length of intubation. Less than half (45.8\%) of patients received early $\mathrm{CHG}$ application, and most (79.2\%) were intubated in the emergency department (ED), suggesting that VAP prevention measures begin in the ED. Of the patients reviewed, five developed VAP; three occurred in patients who had received oral $\mathrm{CHG}$ within 12 hours of intubation. A CNS-driven collaboration with other
\end{abstract}


disciplines and departments is essential to implement VAP prevention measures and provide comprehensive, quality care. 


\section{Acknowledgements}

I would like to express my sincere gratitude to my "lifesavers," Cynthia Padula, $\mathrm{PhD}$, Joan Walsh RN, MSN, CCNS, and Stephanie Farquhar RN, MSN, CNS. These and all of my mentors have provided me with superior support and guidance throughout this journey and it's hurdles, and I am grateful for all they have taught and done for me. I would also like to specially thank my colleagues, classmates, educators, family, and friends. Your support, motivation, and encouragement are what allowed me to successfully complete my graduate degree. I truly could not have done any of this without you all.

Finally, I must take the time to thank my best friend for all of the love, patience, kindness, support, encouragement, understanding, and everything in between. I could not have come this far on my own. Invincible. 


\section{Table of Contents}

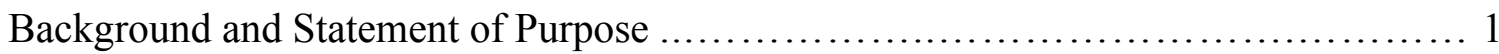

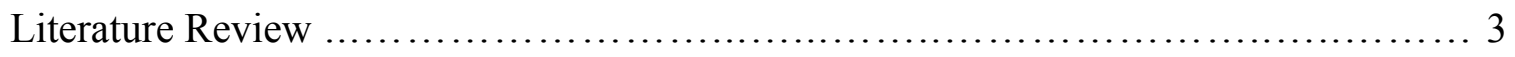

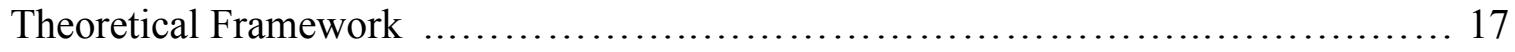

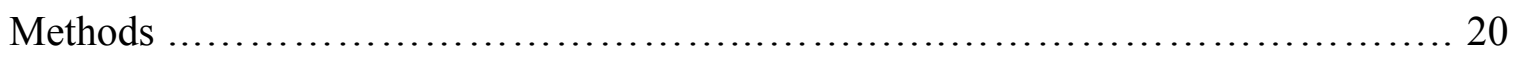

Results .............................................................. 22

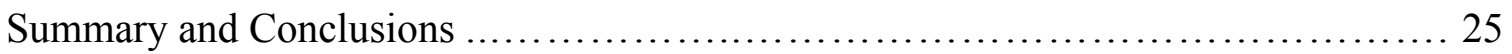

Recommendations and Implications for Advanced Practice Nursing ................ 29

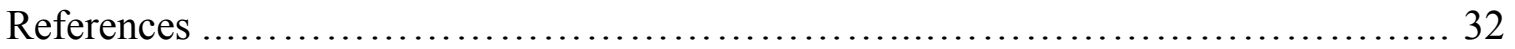

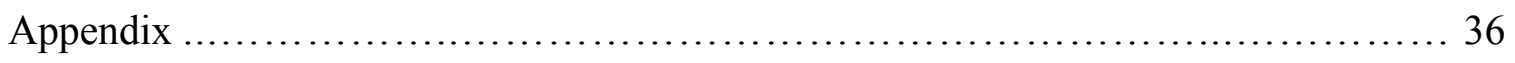


Timeliness of Oral Care and Early-Onset Ventilator Associated Pneumonia Prevention

\section{Background and Statement of Purpose}

Ventilator associated pneumonia (VAP) is a hospital acquired infection defined as a pneumonia occurring in a mechanically ventilated patient that is neither present nor developing upon intubation (Horan, Andrus, \& Dudeck, 2008). The 2004 National Nosocomial Infections Surveillance System (NNIS) report indicated that the greatest VAP incidence is in trauma, burn, neurosurgical, and surgical patients (NNIS, 2004). The VAP rate for fiscal year 2012 in a local Level 1 trauma center, 11-bed trauma intensive care unit was 8.7 per 1000 device days, above the national average of 6 per 1000 device days (NHSN, 2011).

Current practice, guided by the institution's evidence-based oral care protocol for mechanically ventilated patients, includes oral care every four hours with Sage brand oral care kits. Chlorhexidine ( $\mathrm{CHG}) 0.12 \%$ oral solution is administered every 12 hours to the oral cavity including teeth, gums, tongue, and oropharynx. Oral care assessments are conducted daily along with maintaining head of bed elevation $>30$ degrees unless medically contraindicated. As part of the VAP prevention bundle, patients receive stress ulcer and deep vein thrombosis prophylaxis unless medically contraindicated. Readiness to extubate is assessed daily, and sedation is minimized when appropriate.

The purpose of this research was to explore the relationship between the time of insertion of an endotracheal tube and first CHG application and early onset (within 72 hours of intubation) VAP. A secondary purpose was to explore potential evidence for 
revision of the current oral care policy. The risk of VAP begins at the moment of intubation, as should prevention methods. 


\section{Literature Review}

A literature search was performed via PubMed and EBSCO database using the following key terms: ventilator associated pneumonia; oral care; chlorhexidine; VAP and cost; mortality and hospital acquired infection; mortality and VAP. Literature was searched for time period dating from 2005 to 2014. Manual searches for relevant articles were also performed when reviewing the literature.

\section{Hospital Acquired Infections}

Hospital acquired infections (HAIs) are a significant cause of morbidity and mortality in United States (US) hospitals and are associated with increased costs. Using data from the National Nosocomial Infections Surveillance system (NNIS, 2004), National Hospital Discharge Survey (2002), and the American Hospital Association Survey (2000), Klevens and colleagues (2007) estimated an occurrence of over 1.7 million HAIs/year in the US, contributing to 98,987 deaths (Klevens et al.). A report on medical costs associated with HAIs by Scott (2009) examined results from published medical and economic literature, including NNIS reports, and estimated costs for the treatment of HAIs. After adjusting for inflation, it was estimated that, in 2007, HAIs cost up to $\$ 45$ billion in the US (Scott). In accordance with the Deficit Reduction Act of 2005, the Centers for Medicare and Medicaid Services (CMS) ceased reimbursement to hospitals for several complications or comorbidities developed during hospitalization, effective October 1, 2008. The list of complications or comorbidities was based on a list of "never events," preventable conditions not present upon hospital admission, including some HAIs. Common HAIs include urinary tract infections, catheter associated blood 
stream infections, surgical-site infections, and VAP (cms.gov, 2012). Because CMS views VAP as a preventable disease, hospitals may not be reimbursed for costs associated with VAP treatment.

\section{Definition and Diagnosis of Ventilator Associated Pneumonia}

Ventilator associated pneumonia (VAP) is a hospital acquired infection defined as a pneumonia occurring in a mechanically ventilated patient with an onset of 48 hours or more after intubation that is neither present nor developing upon intubation (Horan et al., 2008). Currently, there is no gold standard for diagnosing VAP. In a report from the National Healthcare Safety Network (NHSN) and the Centers for Disease Control and Prevention (CDC), Horan and colleagues (2008) describe a widely accepted algorithm for VAP diagnosis. Ventilator associated pneumonia diagnosis is multifactorial and includes radiologic and clinical signs and symptoms. Ventilator associated pneumonia can be suspected when two or more chest radiographs exhibit new or progressive and persistent infiltrates, consolidation, or cavitation and one or more of the following symptoms: fever greater than 38 degrees Celsius without other recognized cause; leukopenia $(<4,000$ WBC) or leukocytosis $(\geq 12,000 \mathrm{WBC})$; or mental status changes in adults $\geq 70$ years old without other recognized cause. At least two or more of the following symptoms must be present: new onset of purulent sputum; change in character of sputum or increased respiratory secretions; new onset or worsening cough, dyspnea or tachypnea; rales or bronchial breath sounds; increased oxygen requirement, increased ventilator demand or worsening gas exchange (oxygen desaturation, $\mathrm{PaO} 2 / \mathrm{FiO} 2 \leq 240$ ); or positive bronchoalveolar lavage cultures (Horan et al., 2008). 
The Clinical Pulmonary Infection Score (CPIS) is a tool developed to facilitate the diagnosis of VAP. The CPIS consists of six parameters including: temperature; white blood cell count; tracheal secretions; oxygenation; chest radiograph; and tracheal aspirate culture. Each parameter is assigned a point value, with final scores ranging from 0 to 12 , with a score of 6 indicative of VAP. Zilberberg and Shorr (2010), after conducting a meta-analysis, concluded that though some studies suggest a correlation between CPIS $\geq$ 6 and VAP, most studies indicate limited sensitivity and specificity. Additionally, no studies have validated CPIS in acute lung injury or trauma populations. Diagnosis of VAP in trauma patients is further complicated in the presence of blunt chest trauma and pulmonary contusions, as the physiologic response often mimics symptoms of VAP (Zilberberg \& Shorr). Though CPIS is not a reliable diagnostic tool, it may aide clinicians in raising and index of suspicion for VAP.

\section{Pathogenesis}

Pneumonia is caused by microbial invasion of the lower respiratory tract and lung parenchyma resulting from impaired or overwhelmed defense mechanisms. The respiratory tract is comprised of various defense mechanisms to protect the lungs from infection, including: anatomic barriers (glottis, larynx); cough reflexes; mucociliary lining; tracheobronchial secretions; cell-mediated and humoral immunity; and systemic phagocytes involving alveolar macrophages and neutrophils. When these mechanisms fail, microorganisms multiply rapidly. Pathogens may enter the lower respiratory tract via: aspiration, inhalation, translocation, or invasion from distant site. Clinically, VAP may arise from micro-aspiration of oral secretions, aspiration of gastric contents, 
condensate from ventilator tubing, tracheal suctioning, and ventilation with contaminated equipment (Harris \& Tenhet, 2012).

Ventilator associated pneumonia is believed to primarily result from aspiration of oropharyngeal secretions around the endotracheal tube (ETT) cuff into the lungs (Grap, Munro, Unoki, Hamilton, \& Ward, 2012). Oral flora change, often with increasing virulence, within the first 48 hours of critical illness and are hypothesized to contribute to VAP (Bonten et al., 1996). Presence of an ETT impairs natural upper airway defense mechanisms and can create binding sites for microorganisms causing a biofilm, a reservoir of potential pathogens (Grap et al., 2012).

Microorganisms causative of VAP may vary depending on patient population, geographic location, duration of stay, and previous exposure to antimicrobial agents. According to the meta-analysis conducted by Chastre and Fagon (2002), gram-negative bacteria (GNB) have been reported to be causative agents of more than $60 \%$ of VAP cases. Out of 1,689 episodes of VAP and 2,490 pathogens, predominant GNB included: Pseudomonas aeurginosa, 24.4\%; Acinetobacter species (spp.), 7.9\%; Proteus spp., 22.3\%; Escherichia coli, 24.1\%; Klebsiella spp., 15.6\%; and Haemophilus influenzae, 9.8\% (Chastre \& Fagon, 2002). Regarding early-onset VAP, elevated rates of $H$. influenza, Streptococcus pneumoniae, methicillin sensitive Staphylococcus aureus (MSSA) or susceptible Enterobacteriaceae were frequently present. P. aeurginosa, Acinetobacter spp., methicillin resistant Staphylococcus aureus (MRSA), and multiresistant GNB were more frequent in late-onset VAP (Chastre \& Fagon). 


\section{Impact}

Ventilator associated pneumonia is a common hospital-acquired infection that impacts length of stay and hospital costs with the potential to considerably increase mortality rates. Studies of patients with VAP often yield contradictory results, challenging attempts to provide estimates of overall economic and clinical consequences. Though it is impossible to evaluate exact morbidity and excess costs associated with VAP, prolonged hospitalization related to VAP has been estimated in several studies (Chastre \& Fagon, 2002).

The 2004 National Nosocomial Infections Surveillance System Report indicates that the greatest VAP incidence is in trauma, burn, neurosurgical and surgical patients (NNIS, 2004). According to the NHSN 2010 report, VAP incidence amongst adult critical care units ranged from 1.1 to 7.4 per 1,000 patient days and in trauma patients 6.5 per 1,000 patient days (NHSN, 2010).

A retrospective matched cohort study that analyzed the economic burden of VAP in 54 medical centers found that each case cost an average of $\$ 32,140$. The average duration of intubation was 10.1 days in patients with VAP versus 4.7 days for control patients and average duration of hospitalization was 26.5 days vs. 14 days in the control group (Restrepo et al., 2010).

In a secondary analysis of a multicenter prospective cohort study in twenty-seven European ICUs, Blot et al. (2013) investigated the epidemiology of VAP in middle-aged (45-64 years, $n=670)$; old $(65-74$ years, $n=549)$; and very old $(\geq 75$ years, $n=516)$ ICU 
patients. They found that VAP occurred in $14.6 \%$ of middle-aged, $17 \%$ of old, and $12.8 \%$ of very old patients. Though older age was not demonstrated as a risk factor for developing VAP, mortality among patients with VAP was higher in older patients (old and very old, $51 \%)$ than that of middle-aged patients (35\%) (Blot et al.).

A meta-analysis by Chastre \& Fagon (2002) analyzed and described the epidemiology of VAP including: incidence; mortality; morbidity; cost; etiologic agents; and pathogenesis. The majority of reports found VAP frequencies between 8 and $28 \%$. In a Nationwide American study, pneumonia rates were 21 times higher for patients who received mechanical ventilation than those who did not. Patients who developed VAP were twice as likely to die as those who did not, and mortality rates were estimated between $24 \%$ and $76 \%$. The available evidence of VAP is associated with a 20 to $30 \%$ higher risk of death than the risk due to the underlying disease process (Chastre \& Fagon, 2002). A retrospective case-control analysis to describe the epidemiology of nosocomial pneumonia in trauma patients and its impact found that, in a subset of 124 trauma patients, $67 \%$ were mechanically ventilated and early-onset pneumonia represented $63 \%$ of the 41 pulmonary infections (Baker, Meredith \& Haponik, 1996).

Upon summary of available data, Chastre \& Fagon (2002) determined that VAP might potentially extend length of stay in the ICU by at least four days, contributing to an increased financial burden. In 1996, a study by Baker and colleagues estimated extraneous costs attributed to nosocomial pneumonia in trauma patients to be US\$40,000. 


\section{Prevention}

Decreasing VAP was one of the interventions included in the Institute for Healthcare Improvement's (IHI) 5 Million Lives Campaign (2012). This campaign is a national initiative to improve patient care and prevent avoidable deaths in hospitals. The IHI supports the use of bundles, groups of disease specific evidence-based interventions used together to optimize patient care and outcomes. The ventilator bundle includes: elevation of the head of bed between 30 and 45 degrees; daily sedation interruptions; daily assessment of readiness to extubate; stress ulcer prophylaxis; deep vein thrombosis prophylaxis; and oral care which includes CHG 0.12\% (IHI, 2012).

\section{Chlorhexidine and Oral Care: Meta-Analyses}

Current literature supports oral care with $\mathrm{CHG}$ as a cost effective measure to prevent VAP. A meta-analysis by Pineda and colleagues (2006) of four randomized control trials of 1202 patients examined the effect of oral care with CHG on VAP incidence in mechanically ventilated patients in mixed medical, surgical, and cardiothoracic ICUs. Two trials used $0.12 \%$ CHG oral rinse twice daily and two used $0.2 \%$ CHG gel three times daily. The overall incidence of VAP in the CHG treated group was $4 \%$ compared to $7 \%$ in the control group, yielding no statistical significance in VAP reduction (Pineda, Saliba \& Solh, 2006).

Chan and colleagues' meta-analysis included 11 RCTs, including 3242 patients in medical, surgical, medical-surgical, trauma, and cardiothoracic ICUs. Four trials assessed the effectiveness of antibiotic oral decontamination and seven evaluated the effectiveness of antiseptic oral decontamination using varying frequency and strengths of 
CHG $(0.12 \%, 0.2 \%$, and $2 \%)$ and povidone-iodine. Nine studies compared treatment with placebo and two used standard oral care as the control. In all but five trials, the prophylactic regimen was given until extubation. Though few trials reported multiple interventions to prevent VAP, three trials mentioned the use of semi-recumbent positioning in conjunction with oral care. Diagnostic criteria for VAP varied across different trials. Three trials required quantitative bronchoalveolar lavage fluid, two used quantitative cultures of bronchoalveolar lavage fluid or endotracheal aspirate, and one used quantitative cultures of tracheal aspirates. The other trials used semi-quantitative techniques or did not require microbiological confirmation. The four trials testing antibiotic oral decontamination did not show a statistically significant reduction in VAP rates. Upon further review, patients receiving oral decontamination with $\mathrm{CHG}$ experienced a significant reduction in VAP incidence (RR: 0.56, 95\% CI: 0.39-0.81). Overall, neither antibiotic nor antiseptic oral decontamination influenced mortality, duration of mechanical ventilation, or duration of ICU stay (Chan et al.)

Chlebicki and Safdar (2007) reviewed seven trials, three included in the work of $\mathrm{Li}$ and colleagues and four additional, which involved topical application of CHG. Of the 1650 enrolled patients, 812 received $\mathrm{CHG}, 512$ received placebo, and 35 received standard oral care. Two studies were conducted in cardiothoracic ICUs, accounting for $55.4 \%$ of the population, and the remaining five studies were conducted in medical or medical-surgical ICUs. In the two trials in cardiothoracic ICU patients, the intervention was implemented before elective endotracheal intubation and was continued until extubation, discharge from the ICU, or death. In the remaining studies, the intervention 
was continued until extubation or for the length of ICU stay. In two trials, $15 \mathrm{~mL}$ of $0.12 \% \mathrm{CHG}$ was applied to the buccal pharyngeal, gingival, tongue, and tooth surfaces for 30 seconds twice daily. One study used a $0.2 \%$ CHG gel applied three times daily to the dental and gingival surfaces. Another study used a $0.2 \% \mathrm{CHG}$ oral rinse but did not specify frequency. Other studies used a $2 \%$ CHG application every six hours, and one used a single application of $0.12 \%$ CHG spray or swab. In the studies including cardiac surgery patients, patients received perioperative antibiotics, and in remaining studies a large proportion of ICU patients received systemic antibiotic treatment in varying type and duration. The incidence of VAP in the control groups of patients in cardiothoracic ICUs was lower than those in the trials conducted in medical-surgical ICUs $(3.82 \% \mathrm{vs}$. 22.07\%). In two trials, oropharyngeal $\mathrm{CHG}$ did not result in a reduction in VAP incidence. In five trials, $\mathrm{CHG}$ was associated with a beneficial effect for VAP prevention; however, in two trials the decrease was not significantly significant. One study found a statistically significant reduction in VAP only in patients intubated for $>24$ hours. Overall, $9.11 \%$ of patients developed VAP in the treatment group vs. $11.81 \%$ in the comparator group. After combining the results of the seven RCTs, a 30\% relative reduction in VAP was reported, although reduction in mortality was not demonstrated. In two trials including cardiac surgical patients, the benefit of CHG was apparent as evidenced by a 59\% relative risk reduction in VAP (Chlebicki \& Safdar).

A meta-analysis and randomized control trial by Tantipong, Morkchareonpong, Jaiyindee \& Thamlikitkul (2008) analyzed the impact of CHG 2\% in VAP prevention, hypothesizing that a higher concentration of CHG may be more effective. The RCT 
enrolled 207 adult patients in ICU and general medical wards receiving mechanical ventilation. Eligible patients were randomized to the $\mathrm{CHG}$ treatment or normal saline groups. Patients in the intervention group received oral care four times daily which included teeth brushing, suctioning of oral secretions, and rubbing the oral mucosa with CHG 2\% gel until extubation. Each patient was assessed daily for the presence of VAP by diagnostic criteria including new, persistent, or progressive infiltrate on chest radiograph in combination with at least three of the following: temperature greater than 38 degrees $\mathrm{C}$ or less than 35.5 degrees $\mathrm{C}$; leukocytosis or leukopenia; and/or purulent tracheal aspirate or positive culture of tracheal aspirate. The incidence of VAP in the intervention group was $4.9 \%$ versus $11.4 \%$ for the control group, suggesting $2 \% \mathrm{CHG}$ as an effective measure for VAP prevention. No differences in mortality were demonstrated. Though not formally analyzed, the authors reported the use of $\mathrm{CHG} 2 \%$ as cost-effective, with the mean cost per patient calculated as 10 times less than the cost of antibiotics necessary to treat a single occurrence of VAP (Tantipong et al., 2008).

A systematic review examined eight randomized control trials to investigate the efficacy of oral CHG versus power tooth brushing and placebo in the prevention of VAP in adult mechanically ventilated patients in medical (1 RCT), medical-surgical (4RCTs), trauma ( $1 R C T)$, and cardiothoracic (2 RCTs) ICUs where mechanically ventilated patients depended on nursing care to meet their oral care needs. Varying concentrations of $\mathrm{CHG}$ were used: four studies used $0.12 \% \mathrm{CHG}$, two used $0.2 \% \mathrm{CHG}$ and two used $2 \%$ CHG. In comparison groups, five used placebo, one received power tooth-brushing, one received normal saline oral rinse and one received a phenolic rinse. A 36\% higher 
chance of VAP was noted in the control group compared with the CHG group (RR 0.64, 95\% CI 0.44-0.91), supporting the use of CHG. One study reported a reduction in mortality, but rates in the other studies appeared to be unaffected by CHG. Upon subgroup analysis on varying concentrations of $\mathrm{CHG}, 2 \% \mathrm{CHG}$ demonstrated a more significant effect on VAP incidence with an RR of $0.53(95 \%$ CI $0.31-0.91 ; p=0.63)$, than $0.12 \%$ and $0.2 \% \mathrm{CHG}$, supporting the use of $2 \% \mathrm{CHG}$ in high-risk patients (Snyders \& Khnodowe, 2011).

A meta-analysis by Li, Xie, Li and Yue (2013) analyzed 16 trials including 2399 mechanically ventilated patients in both intensive care units (ICUs) and general medical floors. Twelve trials assessed the use of oral antiseptics, 10 of which used CHG for oral decontamination and two studies applied povidone-iodine. The remaining four trials evaluated the use of topical antibiotics. Eight studies compared intervention with placebo, two used normal saline, five used standard oral care, and one used $0.01 \%$ potassium permanganate solution as controls. Many studies mentioned the use of multiple interventions for VAP prevention including head of bed elevation and stress ulcer prophylaxis. Twelve RCTs reported systemic antibiotic use in both treatment and control groups. Four studies reported comorbidities with no significant difference in comorbidities between control and treatment groups. In a combination of 12 trials, use of topical antiseptics showed significant reduction in VAP. Trials using CHG showed a significant reduction in VAP (RR: 0.71; 95\% CI: 0.54-0.94) however, there was no significant effect on mortality, duration of ventilation, and duration of ICU stay reported (Li et al.). 


\section{Chlorhexidine and Oral Care: Other Studies}

A quality improvement project by Hutchins and colleagues (2009) implemented a ventilator bundle in an effort to decrease VAP rates in a 12 bed ICU and nine-bed coronary care unit. The project was conducted from May 2005 to December 2007. Hospital ICU guidelines for ventilated patients were revised to incorporate the ventilator bundle and oral care. Oral care product representatives provided education to registered nurses, respiratory therapists, and intensivists. Continuing oral care education was provided a year later when an increase in VAP rates was noted. All mechanically ventilated patients were included in the project unless there was a contraindication to implementing the ventilator bundle. Consistent with IHI recommendations, the bundle included daily sedation breaks, daily assessment of readiness to extubate, peptic ulcer and deep vein thrombosis prophylaxis, and head of bed elevation greater than 30 degrees. Oral care included tooth-brushing twice daily with cetylpyridinium chloride, which was switched to $0.12 \%$ CHG in January 2007 , in addition to oral care with a hydrogen peroxide solution followed by oral moisturizer every four hours. Oropharyngeal suctioning was performed as needed and before position changes, endotracheal tube cuff deflation, or endotracheal tube repositioning. Family members were also educated to the ventilator bundle and oral care practices. Initial compliance was less consistent for the first year of implementation, ranging from $67 \%$ to $100 \%$ during 2005 and improved to $87 \%$ to $100 \%$ in 2006 . After re-education and time, compliance further improved to $91 \%$ to $100 \%$ for 2007 and 2008. As a result of VAP bundle implementation and CHG application, VAP rates decreased by $89.7 \%$ overall from 2004 to 2007, including a rapid 
decrease in 2005 shortly after initial implementation, suggesting that oral care with CHG and a ventilator bundle may be beneficial in preventing VAP (Hutchins et al.).

A randomized control trial by Grap et al. (2011) tested early (within 12 hours of intubation) application of CHG on oral microbial flora and VAP in trauma patients. Patients intubated in the emergency department, in the field, or en route to the emergency department were eligible if enrolled within 12 hours or intubation. Exclusion criteria included previous endotracheal intubation in the last 48 hours, diagnosis of pneumonia at time of intubation, burn injuries, or edentulous patients. A $5 \mathrm{~mL}$ dose of $0.12 \% \mathrm{CHG}$ solution was applied to the oral cavity by swab. Using a standardized protocol tested in a pilot study, all areas of the oral cavity were covered including anterior and posterior pharynx, gums, teeth, tongue, and buccal mucosa. The control group received standard endotracheal intubation and oral care that did not include CHG. After intubation, nursing staff provided standard oral comfort care for control and intervention groups.

Development of VAP was measured by the use of the Clinical Pulmonary Infection Score (CPIS), which consists of the following parameters: temperature; white blood cell count; tracheal secretions; oxygenation; chest radiograph; and tracheal aspirate culture. Scores range from 0 to 12, with VAP diagnosis made with a score of $\geq 6$. Baseline data, including CPIS, were collected at the time of enrollment. Patients were enrolled for 72 hours after intubation or until extubation. Components of the CPIS were collected upon admission to the study and at 48 and 72 hours after enrollment. Of the 145 randomized patients, 71 and 74 were randomized to intervention and control groups, respectively. No significant differences were noted between groups for clinical characteristics except for a 
greater number of male patients in the intervention group. In subjects without pneumonia at baseline, $55.6 \%$ of the control group developed VAP by 48 or 72 hours versus $33.3 \%$ of the intervention group. Based on their findings, the authors suggest that $\mathrm{CHG}$ application closer to the time of intubation may reduce VAP rates in trauma patients (Grap et al.).

Ventilator associated pneumonia is a common hospital-acquired infection that impacts length of stay, hospital costs, and potentially considerable mortality rate. Studies of patients with VAP often yield contradictory results, challenging attempts to provide estimates of overall economic and clinical consequences. Though oral care with $\mathrm{CHG}$ has been demonstrated to reduce VAP, frequency and dosing guidelines remain unclear.

When implementing changes in practice to improve quality care, it is important to consider processes and factors that influence change within a population. 


\section{Theoretical Framework}

Though evidence based practice (EBP) is widely accepted to improve patient outcomes, gaps between EBP and current practice still exist. Because a change in policy or practice requires a change in behavior, it is important to understand the elements of change throughout the implementation process to optimize success.

Rogers' Diffusion of Innovations (1995) theoretical framework is helpful in determining the adoption of an innovation or behavior. Rogers states that there are five elements of an innovation that contribute to the adoption of the innovation. These elements include relative advantage, compatibility, complexity, trialability and observability. Relative advantage is the degree to which the innovation is improved over what is currently being used. It can be influenced by objective data, including cost effectiveness and patient benefit, as well as perceptions of convenience. Compatibility is the degree to which an innovation is perceived as being consistent with the values, experiences, and needs of potential adopters. In order to be successful, an innovation must address a perceived problem. Complexity measures the degree to which an innovation is perceived as difficult to use and understand. If an innovation is simple and well defined, it is often more quickly adopted. Trialability is the degree to which an innovation may be trialed or modified. An innovation that is trialable decreases uncertainty as it allows the clinician to explore its implementation. Observability is the degree to which the results of an innovation are visible to others. Visible results decrease uncertainty and stimulate peer discussions about the innovation and create positive or negative reactions (Rogers, 1995). 
Channels of communication used to disseminate information about an innovation include research publications, databases, mass media, and lectures. Face to face communication is suggested to be the most effective communication strategy. It provides an opportunity to tailor the delivery of information to recipients and discussion of the proposed change. Adoption of an innovation involves risk and uncertainty. Discussion with those who have adopted successfully often provides reassurance and credibility to the innovation (Rogers, 1995).

Rogers describes five categories of adopters: innovators; early adopters; early majority; late majority; and laggards. Innovators are the first individuals to adopt an innovation. They are often willing to take risks, young in age, in higher social and financial classes, and have close contact to scientific sources and other innovators. Early adopters are the second fastest in adopting an innovation and have a high degree of opinion leadership. They are also typically younger in age and of high social and financial status. Early adopters are more discrete and judicious in adoption choices than innovators. The early majority is composed of individuals who adopt an innovation after a varying degree of time. They tend to be slower to decide, have above average social status, and contact with early adopters. Individuals in the late majority approach an innovation with a high degree of skepticism and adopt after the majority of society has adopted. Laggards are the last group to adopt an innovation. These individuals have an aversion to change and are typically tradition focused. They are often older in age, have low social and financial status, and in contact with only family and close friends (Rogers, 1995). 
Rogers' diffusion model describes five steps in the decision making process: knowledge; persuasion; decision; implementation and confirmation. In the knowledge phase, the individual is exposed to an innovation. The persuasion stage begins when the individual seeks information and detail about the innovation. When at the third stage of decision, an individual weighs the advantages and disadvantages of the innovation and decides to either adopt or reject the innovation. An individual employs the innovation and determines its usefulness in the implementation stage. In the confirmation stage, the individual finalizes his/her decision to continue using the innovation. Individuals progress through the decision process at different rates, depending on their category of adopter (Rogers, 1995).

The Diffusion of Innovations theory offers a reasonable explanation for the adoption or rejection of change across a social system. When pursuing the implementation of a change in behavior or new innovation, it is crucial to be cognizant of the elements of change theory. 


\section{Methods}

\section{Purpose}

The purpose of this research was to explore the relationship between the time of insertion of an endotracheal tube and first CHG application and early onset (within 72 hours of intubation) VAP. A secondary purpose was to explore potential evidence for revision of the current oral care policy.

\section{Design}

A retrospective chart review was conducted to explore the relationship between time of insertion of an endotracheal tube, first application of $\mathrm{CHG}$, and onset of VAP in trauma patients.

\section{Sample and site}

The sample included randomly selected adult intubated trauma patients who had been hospitalized on the trauma intensive care unit during the 2012 fiscal year (October 2011 through September 2012). Inclusion criteria included intubated adult trauma patients on the trauma intensive care unit from the 2012 fiscal year with $\mathrm{CHG}$ oral care compliance of $>80 \%$. This was based on percent of oral care documented in the electronic medication record in the 72 hours post intubation. Exclusion criteria included age $<18$ years, burn injury, patients requiring re-intubation, patients with VAP diagnosis greater than 72 hours after intubation, patients intubated less than 48 hours, or patients with a documented aspiration.

The research was conducted at Rhode Island Hospital, a 719 bed non-profit Level 1 trauma center and part of the Lifespan system. 


\section{Procedures}

After IRB approval was obtained, the trauma registrar was contacted with the request to generate a list of intubated trauma patients who had been hospitalized on the trauma intensive care unit during fiscal year 2012. The medical records of potential subjects were reviewed for inclusion and exclusion criteria, and records that met the inclusion criteria were further reviewed for study data. Of the 101 records reviewed, 48 met inclusion criteria for study.

\section{Measurement}

A researcher developed data collection tool (Appendix A) was used to collect the following data: patient age, gender, primary diagnosis, location, date and time of intubation, date and time of first CHG application, date and time of endotracheal tube removal, $\mathrm{CHG}$ oral care provided during the first 72 hours post intubation, and date and time of VAP diagnosis if present.

\section{Data Analysis}

Descriptive statistics were performed on all of the study data. Length of intubation was calculated based on date and time of insertion and date and time of endotracheal tube removal. Percent compliance was calculated based on oral care delivered in the 72 hours post intubation. Timing of CHG application was calculated based on time of intubation and time of first CHG application. 


\section{Results}

Of the 101 charts reviewed, 48 met the inclusion criteria, and consisted of 32 males and 16 females. Four identifiable categories of reason for ED admission were noted including: motor vehicle collision $(\mathrm{n}=21 ; 43.75 \%)$; fall $(\mathrm{n}=11 ; 22.91 \%)$;

motorcycle collision $(n=8 ; 16.67 \%)$; and other $(n=8 ; 16.67 \%)$. Table 1 illustrates data specific to age, days of intubation, and timing of first CHG application.

\section{Table 1}

Selected Demographic Data

\begin{tabular}{|c|c|c|c|}
\hline & Low & High & Average \\
\hline Age & 20 & 93 & 48.7 \\
\hline \# of days intubated & 3 & 26 & 9.45 \\
\hline Timing of CHG & 58 minutes & 67 hours & 18.96 hours \\
First Application & & & \\
& & & \\
\hline
\end{tabular}

In calculating the average number of days intubated, there was one outlier with an intubation time of 121 days. As seen in Table 1, there was a very wide range of times regarding timing of first CHG application, the average almost 19 hours after intubation. Table 2 illustrates the number and percentage of patients intubated by location. 
Table 2

Intubations by Location

\begin{tabular}{|c|c|c|}
\hline $\begin{array}{c}\text { Location of } \\
\text { Intubation }\end{array}$ & $\#$ of patients & $\%$ of patients \\
\hline $\begin{array}{c}\text { Emergency } \\
\text { Department } \\
\text { (ED) }\end{array}$ & 38 & $79.2 \%$ \\
\hline $\begin{array}{c}\text { Operating } \\
\text { Room (OR) }\end{array}$ & 4 & $8.2 \%$ \\
\hline $\begin{array}{c}\text { Trauma } \\
\text { Intensive Care } \\
\text { Unit (TICU) }\end{array}$ & 3 & $6.3 \%$ \\
\hline $\begin{array}{c}\text { Referring } \\
\text { Hospital (RH) }\end{array}$ & 3 & $6.3 \%$ \\
\hline
\end{tabular}

The primary location for intubation was the ED (79.2\%), with the remaining $20.8 \%$ being intubated outside of the ED. Approximately $6 \%$ were intubated at an outside facility, prior to transfer to the study site.

Table 3 illustrates the timing of $\mathrm{CHG}$ application from the time of intubation and the timing of VAP onset in the 48 patients.

\section{Table 3}

Timing of CHG Application from time of Intubation $(n=48)$

\begin{tabular}{|c|c|c|c|}
\hline Timing of CHG & $\leq 12 \mathrm{hr}$ to $\mathrm{CHG}$ & $\begin{array}{c}12 \mathrm{hr} 1 \mathrm{~min} \text { to } 24 \mathrm{hr} \\
\text { to } \mathrm{CHG}\end{array}$ & $>24 \mathrm{hr} \mathrm{CHG}$ \\
\hline \# of patients & 22 & 13 & 13 \\
\hline \% of patients & $45.8 \%$ & $27.1 \%$ & $27.1 \%$ \\
\hline + VAP diagnoses & 3 & 1 & 1 \\
\hline \multicolumn{4}{|r}{$\mathrm{hr}=$ hours; min = minutes }
\end{tabular}

There were five VAP cases identified in the review. Of the patients who received early $\mathrm{CHG}$ application, three were positive for VAP. Of the three, one case of early- 
onset VAP developed at 24 hours 10 minutes after intubation. The remaining two cases developed late, 6 days after intubation. One patient who received oral CHG from 12 to 24 hours after intubation developed VAP, and one patient who received their CHG $>24$ hours after intubation, experienced early-onset VAP.

Next, summary and conclusions will be discussed. 


\section{Summary and Conclusions}

Hospital-acquired infections (HAIs), including ventilator associated pneumonia (VAP), are a significant cause of morbidity and mortality in United States (US) hospitals and are associated with increased costs and length of stay (Chastre \& Fagon, 2002; NNIS, 2004). Ventilator associated pneumonia is described as a pneumonia occurring in a mechanically ventilated patient that is neither present nor developing upon intubation (Horan et al., 2008) and is believed to primarily result from aspiration of oropharyngeal secretions around the endotracheal tube (ETT) cuff into the lungs (Grap et al., 2012). The Institute for Healthcare Improvement (IHI) supports the use of bundles, to optimize patient care and outcomes. The ventilator bundle includes: elevation of the head of bed between 30 and 45 degrees; daily sedation interruptions; daily assessment of readiness to extubate; stress ulcer prophylaxis; deep vein thrombosis prophylaxis; and oral care which includes CHG 0.12\% (IHI, 2012).

Current practice, guided by the institution's evidence-based oral care protocol for mechanically ventilated patients, included oral care every four hours with Sage brand oral care kits. Chlorhexidine (CHG) $0.12 \%$ oral solution is administered every 12 hours to the oral cavity including teeth, gums, tongue, and oropharynx. Oral care assessments are conducted daily along with maintaining head of bed elevation $\geq 30$ degrees unless medically contraindicated. As part of the VAP prevention bundle, patients receive stress ulcer and deep vein thrombosis prophylaxis unless medically contraindicated. Readiness to extubate is assessed daily, and sedation is minimized when appropriate. 
The purpose of the study was to explore the relationship between the time of insertion of an endotracheal tube, the first CHG application and early onset (within 72 hours of intubation). A retrospective chart review was conducted by reviewing the records of randomly selected adult intubated trauma patients who had been hospitalized on the trauma intensive care unit (TICU) during the 2012 fiscal year (October 2011 September 2012) and who met additional inclusion criteria

Though the evidence and policy recommended administration of CHG within 12 hours of intubation, less than half (45.8\%) of patients received CHG within 12 hours of intubation, the average time to CHG application was 18.96 hours. Of the patients who received early CHG application, three developed VAP. One case of early-onset VAP developed at 24 hours 10 minutes after intubation, and 2 additional cases developed late, 6 days after intubation. One patient who received oral CHG from 12 to 24 hours after intubation developed VAP, and one patient who received $\mathrm{CHG}>24$ hours after intubation experienced early-onset VAP. To the researcher's surprise, there were more diagnoses of VAP in patients who received oral CHG within 12 hours of intubation than those who received oral CHG application late after intubation, for which there was no clear explanation.

Limitations to the study included incomplete or missing records and limited numbers due to the criteria excluding patients with a diagnosis of burn and those admitted to the neurosurgical intensive care unit. Also, there was no attempt to examine the relationship of severity of diagnosis and other key variables that may have contributed to VAP risk factors. 
Most patients (79.2\%) were intubated in the ED of the admitting hospital, suggesting that there is an opportunity to advocate for oral care practices to begin in the emergency department. A randomized control trial by Grap et al. (2011) tested early (within 12 hours of intubation) application of CHG on oral microbial flora and VAP in trauma patients. In subjects without pneumonia at baseline, $55.6 \%$ of the control group developed VAP by 48 or 72 hours versus $33.3 \%$ of the intervention group. Based on their findings, the authors suggested that $\mathrm{CHG}$ application closer to the time of intubation might reduce VAP rates in trauma patients (Grap et al., 2011).

In fiscal year 2013, VAP rates in the TICU decreased from 8.7 to 5.8 per 1000 device days, a $28 \%$ decrease in frequency after a quality improvement project, unrelated to this research, was conducted regarding appropriate oral care practices. This data suggests that increased awareness and education may improve infection control practices, contributing to a culture of safety.

There is a need for improvement in training and compliance with oral care policies to ensure the delivery of effective, quality care throughout hospitalization. Institutional policies should reflect current, evidence-based recommendations including early administration of CHG after intubation to decrease VAP occurrence, improve patient outcomes and decrease costs. Though there may not be strong evidence to link early $\mathrm{CHG}$ administration to decreased infection rates, implementation of $\mathrm{CHG}$ administration immediately following intubation in the ED is recommended since the cost of the intervention is low, side effects have not been identified, and its potential impact is high. 
Next, recommendations for advanced practice nursing will be discussed. 


\section{Recommendations and Implications for Advanced Nursing Practice}

Advanced Practice Registered Nurses (APRNs) are integral to improving quality care and outcomes for patients, families, and communities. As leaders, APRNs must role model best practices and maintain organizational involvement to identify needs and priorities of the system at all levels.

The work of a Clinical Nurse Specialist (CNS) is based in three spheres of influence: patients/families, nurses/nursing practice, and organization/systems. As a change agent, the CNS uses evidence-based practice (EBP) to develop, implement, and evaluate initiatives for improving quality care and creating a culture of safety. Oral care and other policies should be regularly updated and implemented to ensure they are reflective of current evidence-based practices. In creating a culture of safety, it is crucial to lead by example when implementing and advocating for appropriate infection control measures and to encourage peer-to-peer accountability for compliance to quality care measures.

Though oral care with CHG has been demonstrated to reduce VAP, frequency and dosing guidelines remain unclear and further investigation is needed. The CNS is critical to keeping practitioners abreast of evidence-based practice, conducting quality improvement and research projects, and disseminating this information to bedside nurses in a way that is meaningful to their practice. This may be achieved by linking quality measures to care compliance, cost, and comparison to similar facilities.

The use of technology could be beneficial in ensuring the delivery of quality care. 
Simulation and hands-on learning for providing appropriate, effective mouth care to intubated patients may improve oral care practices. High-risk infection prevention competencies should be included regularly in nurse credentialing or education sessions to reinforce up to date infection control practices. The CNS can collaborate with the institution's internal technology department and pharmacy department to create order sets and facilitate access to $\mathrm{CHG}$ to reduce barriers to timely application.

A CNS-driven collaboration with other disciplines is imperative in providing comprehensive, quality care. Participation in professional organizations is essential for networking and information sharing. Involvement of the CNS in professional practice organizations invites the opportunity to advocate for policies that are evidence based at the local, state, and national level. Clinical nurse specialists can make contributions to evidence-based literature, through the utilization of their advanced skills in researching, implementing, and evaluating evidence-based practice.

An interdisciplinary approach to VAP prevention including nurses, respiratory therapists, physical/occupational therapists, nutritionists, and others, is required. Interdisciplinary interventions encompass the patient as a whole to meet his/her individual needs in the pursuit of quality care.

Outcome measures have become a top priority in healthcare related to reimbursement rates and penalty fees. Practitioners at all levels are responsible for demonstrating delivery of quality, evidence-based care delivery to improve patient outcomes and prevent hospital acquired conditions and infections. The CNS has great 
influence in primary prevention, population management, and improving nurse-sensitive outcome measures.

Further research in VAP prevention is needed, with more controlled studies focusing on specialty populations. Study in multi-trauma populations should consider other variables associated with trauma including co-morbidities, severity, and anatomic location of injury.

The expertise of the CNS can prove invaluable in policy development, practice changes, improving nurse sensitive outcomes, and improving organizational processes to optimize the delivery of quality, patient-centered care for safe passage through the healthcare system. 


\section{References}

Baker, A., Meredith, J., \& Haponik, E. (1996). Pneumonia in intubated trauma patients: Microbiology and outcomes. American Journal of Respiratory Critical Care Medicine, 153, 343-349.

Blot, S., Koulenti, D., Dimopoulos, G., Komnos, A., Kreuger, W., Spina, G., et al. (2013). Prevalence, risk factors, and mortality for ventilator-associated pneumonia in middle-aged, old, and very old critically ill patients. Critial Care Medicine, 42(3), 601-609.

Bonten, M., Bergmans, D., Ambergen, A., Leeuw, P. d., der Geest, S. V., Stobberingh, E., et al. (1996). Risk factors for pneumonia, and colonization of respiratory tract and stomach in mechanically ventilated ICU patients. American Journal of Respiratory Critical Care Medicine, 154, 1339-1346.

Chan, E., Ruest, A., Meade, M., \& Cook, D. (2007). Oral decontamination for prevention of pneumonia in mechanically ventilated adults: systematic review and meta analysis. British Medical Journal, 334. Retrieved June 22, 2013, from http://www.ncbi.nlm.nih.gov/pmc/articles/PMC1857782/

Chastre, J. \& Fagon, J. (2002). Ventilator-associated pneumonia. American Journal of Respiratory Critical Care Medicine, 165, 867-903.

Chlebicki, M. \& Safdar, N. (2007). Topical chlorhexidine for prevention of ventilatorassociated pneumonia: A meta-analysis. Critical Care Medicine, 35(2), 595-602. 
cms.gov. (2012, September 20). Hospital-acquired conditions - Centers for Medicare \& Medicaid Services. Centers for Medicare \& Medicaid Services . Retrieved August 14, 2013, from http://www.cms.gov/Medicare/Medicare-Fee-for-ServicePayment/HospitalAcqCond/Hospital-Acquired_Conditions.html

Grap, M., Munro, C., Unoki, T., Hamilton, V., \& Ward, K. (2012). Ventilator-associated pneumonia: the potential critical role or emergency medicine in prevention. The Journal Of Emergency Medicine, 42(3), 353-362.

Grap, M. J., Munro, C., Hamilton, V., Elswick, R., Sessler, C., \& Ward, K. (2011). Early, single chlorhexidine application reduces ventilator associated pneumonia in trauma patients. Heart \& Lung, 40, 115-112.

Harris, J. \& Tenhet, M. (2012). Common Respiratory Disorders. Critical Care Nursing: A Holistic Approach (10 ed., pp. 566-607). Philadelphia: Lippincott Williams \& Wilkins.

Horan, T., Andrus, M., \& Dudeck, M. (2008). CDC/NHSN surveillance definition of health care-associated infection and criteria for specific types of infections in the acute care setting. American Journal of Infection Control, 36(5), 309-332.

Hutchins, K., Karras, G., Erwin, E., \& Sullivan, K. (2009). Ventilator associated pneumonia and oral care: a successful quality improvement project. American Journal of Infection Control, 37(7), 590-597.

Institute for Healthcare Improvement (IHI) (2012). Implement the IHI Ventilator Bundle. Retrieved September 13, 2013, from http://www.ihi.org/resources/Pages/Changes/ImplementtheVentilatorBundle.aspx 
Klevens, M., Edwards, J., Richards, C., Horan, T., Gaynes, R., Pollock, D., et al. (2007). Estimating health care-associated infections and deaths in U.S. hospitals, 2002. Public Health Reports, 122, 160-166.

Li, J., Xie, D., Li, A., \& Yue, J. (2013). Oral topical decontamination for preventing ventilator-associated pneumonia: A systematic review and meta-analysis of randomized controlled trials. Journal of Hospital Infection, 4(12), 1-11.

National Healthcare Safety Network (NHSN) (2011). National Healthcare Safety Network Report. National Center for Emerging and Zoonotic Infectious Diseases, 1, 1-35.

National Healthcare Safety Network (NHSN) (2010). National Healthcare Safety Network Report. National Healthcare Safety Network (NHSN) Report, Data Summary for 2009, Device-associated Module.

NNIS. (2004). National Nosocomial Infections Surveillance (NNIS) System Report, data summary from January 1992 through June 2004, issued October 2004. American Journal of Infection Control, 32, 470-485.

Pineda, L., Saliba, R., \& Solh, A. (2006). Effect of oral decontamination with chlorhexidine on the incidence of nosocomial pneumonia: A meta-analysis. Critical Care, 10. Retrieved June 22, 2013, from http://ccforum.com/content/10/1/R35

Restrepo, M., Anzueto, A., Arroliga, A., Afessa, B., Atkinson, M., Ho, N., et al. (2010). Economic burden of ventilator-associated pneumonia based on total resource utilization. Infection Control and Hospital Epidemiology, 31(5), 509-515. 
Rogers, E. (1995). Diffusion of innovations (4th ed.). New York: The Free Press.

Scott II, R.D. (2009). The direct medical costs of healthcare-associated infections in U.S. hospitals and the benefits of prevention. Retrieved from www.cdc.gov/ncidod/dhqp.pdf/scottcostpaper

Snyders, O. \& Khondowe, O. (2011). Oral chlorhexidine in the prevention of ventilatorassociated pneumonia in critically ill adults in the ICU: A systematic review. Southern African Journal of Critical Care , 27(2), 48-56.

Tantipong, H., Morkchareonpong, C., Jaiyindee, S., \& Thamlikitkul, V. (2008). Randomized controlled trial and meta-analysis of oral decontamination with $2 \%$ chlorhexidine solution for the prevention of ventilator-associated pneumonia. Infection Control and Hospital Epidemiology, 29(2), 131-136.

Zilberberg, M. \& Shorr, A. (2010). Ventilator-associated pneumonia: The clinical pulmonary infection score as a surrogate for diagnostics and outcome. Clinical Infectious Diseases, 51(1), 131-135. 


\section{Appendix A}

\section{Data Collection Tool}

\begin{tabular}{|c|c|c|c|c|c|c|c|c|c|c|c|c|c|}
\hline $\begin{array}{l}\text { Patie } \\
\text { nt } \\
\text { Age }\end{array}$ & $\begin{array}{l}\text { Gend } \\
\text { er }\end{array}$ & \begin{tabular}{|c} 
Primar \\
y \\
Diagn \\
osis \\
\end{tabular} & $\begin{array}{c}\text { Locatio } \\
\mathrm{n} \text { of } \\
\text { Intubati } \\
\text { on }\end{array}$ & $\begin{array}{c}\text { Date of } \\
\text { Intubat } \\
\text { ion }\end{array}$ & $\begin{array}{c}\text { Time } \\
\text { of } \\
\text { Intubati } \\
\text { on }\end{array}$ & $\begin{array}{l}\text { Date } \\
\text { of } 1 \mathrm{st} \\
\mathrm{CHG}\end{array}$ & $\begin{array}{l}\text { Time } \\
\text { of } 1 \mathrm{st} \\
\mathrm{CHG}\end{array}$ & $\begin{array}{l}\text { Date of } \\
\text { Extubati } \\
\text { on }\end{array}$ & $\begin{array}{c}\text { Time } \\
\text { of } \\
\text { Extubat } \\
\text { ion }\end{array}$ & $\begin{array}{c}\text { Length } \\
\text { of } \\
\text { Intubati } \\
\text { on }\end{array}$ & \begin{tabular}{|c|} 
Oral \\
Care \\
Complia \\
nce \\
\end{tabular} & \begin{tabular}{|c|} 
Date and \\
Time of \\
VAP \\
Diagnosis \\
\end{tabular} & \begin{tabular}{|c|} 
Timing \\
of CHG \\
Applicat \\
ion \\
\end{tabular} \\
\hline & & & & & & & & & & & & & \\
\hline & & & & & & & & & & & & & \\
\hline
\end{tabular}

